\title{
Experimental Verification of a Two-Degree-of-Freedom Resonant Actuator Using Its Detent Force
}

\author{
Gyunam Kim $^{* a)}$ Non-member, Katsuhiro Hirata* Senior Member \\ (Manuscript received May 29, 2020, revised Jan. 23, 2021) \\ J-STAGE Advance published date : March 26, 2021
}

\begin{abstract}
This study aims to present a new two-degree-of-freedom resonant actuator and its motion control method. Accordingly, the design method for this resonant actuator that resonates using its detent force without mechanical spring is proposed. In this actuator, a centering force is generated according to the moving distance, regardless of the moving direction on the $X-Y$ plane. Using this feature, various resonance motions on the $X-Y$ plane can be realized. This study also proves that the reciprocating motion, elliptical motion, and scroll motion can be controlled. The results of finite element analysis support the characteristics of the proposed actuator, which were compared to the results of measurements on a prototype. Finally, the effectiveness of motion control was validated through the prototype.
\end{abstract}

Keywords: multi-degree-of-freedom, linear resonant actuator, detent, spring-less, motion control

\section{Introduction}

Recently, various actuators for application to household appliances and industrial machines have been actively studied. Linear resonant actuators (LRAs) are an actuator that reciprocates with high efficiency using $\mathrm{m}-\mathrm{k}$ resonance. LRAs are mainly used for compressors, electric shavers, electric toothbrushes, and artificial hearts ${ }^{(1)-(5)}$. On the other hand, a multiple-degree-of-freedom (M-DOF) resonant actuator (RA) which extends DOF of LRA has also been studied. This M-DOF RA can be used for applications requiring various motions such as a haptic device ${ }^{(6)(7)}$. However, since general M-DOF RA can change the effective air-gap, precise motion cannot be realized due to variations in the motor parameters. Furthermore, as general M-DOF RA uses mechanical spring for resonant operation, the number of parts increases and the overall system becomes bulky and complicated. Also, the M-DOF RA is difficult to realize full resonant operation in a variety of motions due to differences in vertical stiffness and lateral stiffness of the mechanical springs. For example, the two-DOF RA can be designed to have the same resonance frequency in the $X$ and $Y$ directions, but the resonance frequency for some angles may be different. The phase difference between the current and the velocity of the mover is made due to the change of the resonance frequency depending on the moving direction. The phase difference eventually makes it difficult to control the motion. Moreover, since MDOF RA controls two or more coils independently, interference between mutual inductances is large and the estimation error of displacement may accordingly be large. However, designing the axial gap type M-DOF RA with a star or delta connection of three-phase symmetric coils without using a mechanical spring can solve the problems mentioned ${ }^{(8)(9)}$.

a) Correspondence to: Gyunam Kim. E-mail: gyunam.kim@ mapse.eng.osaka-u.ac.jp

* Department of Adaptive Machine System, Osaka University

2-1, Yamadaoka, Suita 565-0871, Japan
$\mathrm{Nd}-\mathrm{Fe}-\mathrm{B}$ permanent magnet was used in the cited actuator. However, it was difficult to design the support structure due to the strong attraction force generated in the $Z$-direction. Thus, we redesigned it by changing as a ferrite permanent magnet.

In this study, we redefine that the detent characteristic is similar to a type of mechanical springs. In addition, the thrust characteristic by phase current is presented. They are verified through finite element analysis (FEA). To control various motion, we propose a simplified motion that can express all of the motion in $X-Y$ plane. As a result, they can be represented as only three types of variables. We also present the prototype of the proposed actuator. All of the static parameters including detent, thrust constant, inductance, and resistance are measured and compared to the results of FEA. Finally, we confirm that the various motion with two-DOF is can be realized by experimental result.

\section{Characteristics of the Proposed Actuator}

2.1 Proposed Actuator Figure 1 demonstrates the

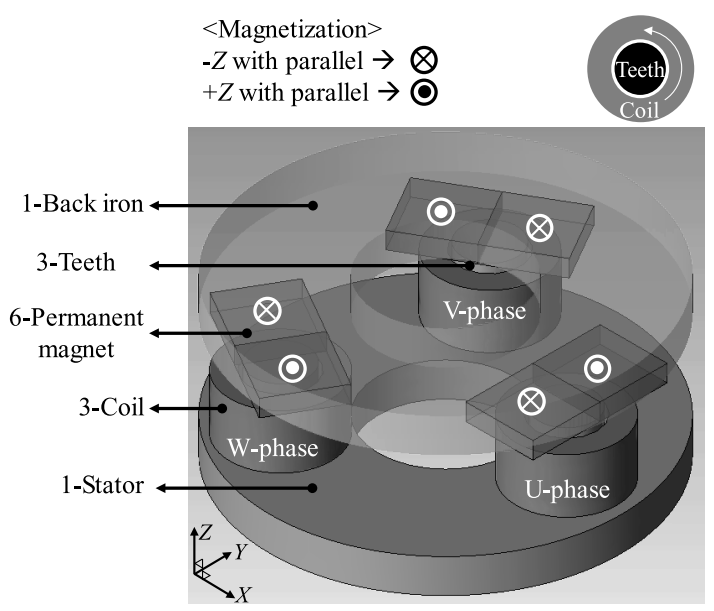

Fig. 1. Basic structure of the proposed two-DOF RA 


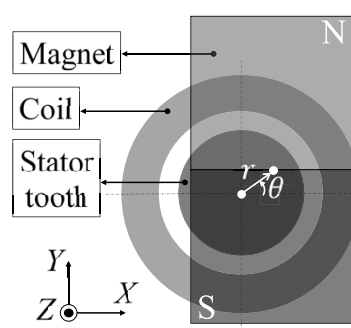

(a)

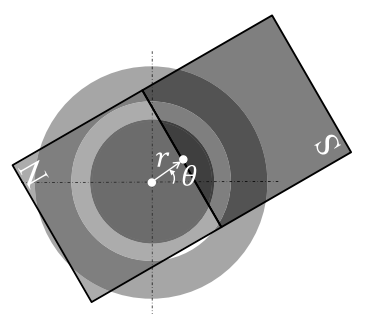

(b)

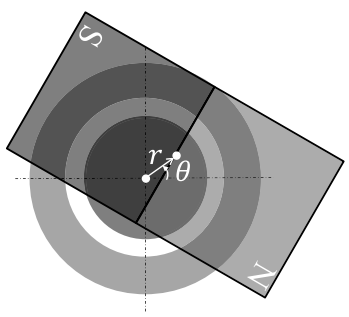

(c)
Fig. 2. Position of the mover and each phase magnet. (a) U-phase (b) V-phase (c) W-phase

basic structure of the proposed two-DOF RA. The actuator consists of stator, coil, permanent magnet, and moving core. The three-phase coils are connected by a star-connection and wound counterclockwise when viewed from the top. Magnets placed on $U-V-W$ phase with $120^{\circ}$ interval are fixed to the back-iron. In addition, three magnets are magnetized in the positive direction of the $Z$-axis while the others are magnetized in the negative direction of the $Z$-axis. Since the mover is constrained only in the $Z$-direction, various resonant motion can be realized on the $X-Y$ plane.

2.2 Detent Characteristics Figure 2 indicates the position of each phase magnet when the mover is placed at the position of a distance $r$ and an angle $\theta$. To define the detent force reacted in each phase magnet, the force vector in each phase frame can be defined as:

$$
\begin{aligned}
\mathbf{F}_{r u} & =\left[\begin{array}{l}
A r \cos \theta \\
B r \sin \theta
\end{array}\right] \ldots \ldots \ldots . \\
\mathbf{F}_{r v} & =\left[\begin{array}{l}
A r \cos \left(\theta-\frac{2}{3} \pi\right) \\
B r \sin \left(\theta-\frac{2}{3} \pi\right)
\end{array}\right] . \\
\mathbf{F}_{r w} & =\left[\begin{array}{l}
A r \cos \left(\theta-\frac{4}{3} \pi\right) \\
B r \sin \left(\theta-\frac{4}{3} \pi\right)
\end{array}\right] .
\end{aligned}
$$

where $\mathbf{F}_{r u}$ means the reaction force vector of magnets placed in U-phase, $\mathbf{F}_{r v}$ denotes the reaction force vector in V-phase, $\mathbf{F}_{r w}$ is the reaction force vector in $\mathrm{W}$-phase, $A$ is a gain in the direction of each phase axis, and $B$ stands for a gain in the normal direction of each phase axis. Since $A$ and $B$ are determined by material and the shape of magnets, Eqs. (1)-(3) are the generalized expression.

The above reaction force vectors are presented in each phase axis and their normal direction. They can be represented by converting to the $X-Y$ orthogonal frame as follows:

$$
\begin{aligned}
& {\left[\begin{array}{l}
F_{d u x} \\
F_{d u y}
\end{array}\right]=\mathbf{R}(0) \mathbf{F}_{r u}=\left[\begin{array}{l}
A r \cos \theta \\
B r \sin \theta
\end{array}\right] \ldots \ldots \ldots \ldots \ldots \ldots} \\
& {\left[\begin{array}{l}
F_{d v x} \\
F_{d v y}
\end{array}\right]=\mathbf{R}\left(-\frac{2}{3} \pi\right) \mathbf{F}_{r v}} \\
& =\left[\begin{array}{l}
1 \\
-\frac{1}{2} A r \cos \left(\theta-\frac{2}{3} \pi\right)-\frac{\sqrt{3}}{2} B r \sin \left(\theta-\frac{2}{3} \pi\right) \\
\frac{\sqrt{3}}{2} A r \cos \left(\theta-\frac{2}{3} \pi\right)-\frac{1}{2} B r \sin \left(\theta-\frac{2}{3} \pi\right)
\end{array}\right] \ldots \\
& {\left[\begin{array}{l}
F_{d w x} \\
F_{d w y}
\end{array}\right]=\mathbf{R}\left(-\frac{4}{3} \pi\right) \mathbf{F}_{r w}} \\
& =\left[\begin{array}{c}
\frac{1}{2} A r \cos \left(\theta-\frac{4}{3} \pi\right)+\frac{\sqrt{3}}{2} B r \sin \left(\theta-\frac{4}{3} \pi\right) \\
-\frac{\sqrt{3}}{2} A r \cos \left(\theta-\frac{4}{3} \pi\right)-\frac{1}{2} B r \sin \left(\theta-\frac{4}{3} \pi\right)
\end{array}\right] \ldots .
\end{aligned}
$$

where $F_{d u x}$ and $F_{d u y}$ present detent force of U-phase magnet in $X-Y$ orthogonal frame, $F_{d v x}$ and $F_{d v y}$ means the detent force of V-phase magnet, $F_{d w x}$ and $F_{d w y}$ are the detent force of W-phase, and $\mathbf{R}$ is Park's transformation matrix.

Since the detent force of the mover is equal to the sum of detent force in each phase magnet, it can be obtained as:

$$
\begin{aligned}
& F_{d x}=F_{d u x}+F_{d v x}+F_{d w x}=\frac{3}{2}(A+B) r \cos \theta \ldots \\
& F_{d y}=F_{d u y}+F_{d v y}+F_{d w y}=\frac{3}{2}(A+B) r \sin \theta \ldots
\end{aligned}
$$

where $F_{d x}$ denotes the detent force of the mover in $X$ direction and $F_{d y}$ means the detent force in $Y$-direction.

In addition, when the mover is placed at the position of the distance $r$ and the angle $\theta$, the detent force in $\theta$ direction and its normal direction can be obtained as follows:

$$
\mathbf{F}_{d \theta}=\mathbf{R}(\theta)\left[\begin{array}{l}
F_{d x} \\
F_{d y}
\end{array}\right]=\left[\begin{array}{c}
\frac{3}{2}(A+B) r \\
0
\end{array}\right] \ldots
$$

where $\mathbf{F}_{d \theta}$ denotes the vector presented as components of $\theta$ direction and its normal direction.

From Eq. (9), since the component of normal direction keeps the value of zero, we know that the mover always received the force in the origin direction wherever it is positioned. This characteristic is similar to the case that several mechanical springs are mounted radially in the $X-Y$ plane.

2.3 Thrust Characteristics As the proposed actuator uses a star-connection, assuming that three-phase currents are in equilibrium as follows:

$$
i_{u}+i_{v}+i_{w}=0
$$

where $i$ denotes current, and subscript means each phase.

Furthermore, as the force constant $K_{f}$ of each phase is all the same, the sum of the torque $T$ generated at each phase becomes 0 as follows:

$$
T=l\left(F_{u}+F_{v}+F_{w}\right)=l K_{f}\left(i_{u}+i_{v}+i_{w}\right)=0 \cdots \cdots
$$

where $l$ is the distance between the center of the stator and the tooth, and $F$ means the force generated in each phase.

Meanwhile, the Park's and Clarke's transformation matrix $\mathbf{T}$ can be defined with magnitude invariance system as follows: 


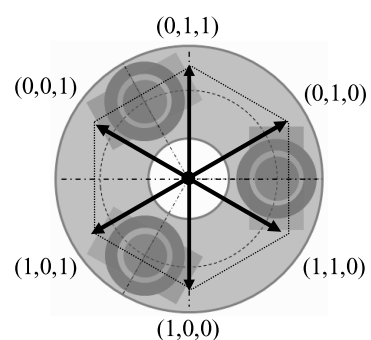

(a)

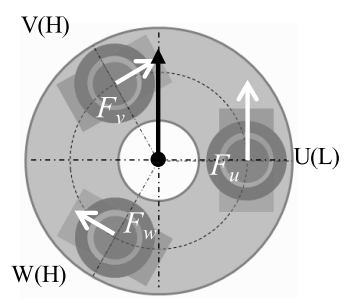

(b)
Fig. 3. Switch states and force direction. (a) force direction in each switch state (b) force direction on $(0,1,1)$

$$
\mathbf{T}(\theta)=\frac{2}{3}\left[\begin{array}{ccc}
\cos \theta & \cos \left(\theta-\frac{2}{3} \pi\right) & \cos \left(\theta+\frac{2}{3} \pi\right) \\
-\sin \theta & -\sin \left(\theta-\frac{2}{3} \pi\right) & -\sin \left(\theta+\frac{2}{3} \pi\right)
\end{array}\right]
$$

By considering the sum of the current force on each phase, the thrust of the mover can be defined as:

$$
\left[\begin{array}{l}
F_{e x} \\
F_{e y}
\end{array}\right]=\mathbf{T}\left(\frac{\pi}{2}\right)\left[\begin{array}{l}
F_{u} \\
F_{v} \\
F_{w}
\end{array}\right]=\frac{2}{3} K_{f}\left[\begin{array}{c}
\frac{\sqrt{3}}{2} i_{v}-\frac{\sqrt{3}}{2} i_{w} \\
-i_{u}+\frac{\sqrt{3}}{2} i_{v}+\frac{\sqrt{3}}{2} i_{w}
\end{array}\right]
$$

where $F_{e x}$ denotes the thrust in the $X$-direction, $F_{e y}$ means the thrust in the $Y$-direction.

2.4 Current Mode and Force Direction By using a three-phase voltage source inverter, current mode can be generated as shown in Fig. 3(a). As shown in Fig. 3(b), the direction of the force according to the current mode is determined by the sum of the vector components of the force generated in each phase. In addition, force components of various directions can be created by a composite vector of two modes.

\section{Model Dynamics}

3.1 Electrical Dynamics The electrical dynamics of the two-DOF RA with three-phase coils can be expressed as follows:

$$
\begin{aligned}
{\left[\begin{array}{c}
v_{u} \\
v_{v} \\
v_{w}
\end{array}\right]=} & {\left[\begin{array}{ccc}
R_{u} & 0 & 0 \\
0 & R_{v} & 0 \\
0 & 0 & R_{w}
\end{array}\right]\left[\begin{array}{c}
i_{u} \\
i_{v} \\
i_{w}
\end{array}\right] } \\
& +\frac{d}{d t}\left\{\left[\begin{array}{lll}
L_{u u} & M_{u v} & M_{u w} \\
M_{v u} & L_{v v} & M_{v w} \\
M_{w u} & M_{w v} & L_{w w}
\end{array}\right]\left[\begin{array}{c}
i_{u} \\
i_{v} \\
i_{w}
\end{array}\right]\right\}+\left[\begin{array}{c}
e_{u} \\
e_{v} \\
e_{w}
\end{array}\right] \ldots \ldots
\end{aligned}
$$

where $v$ and $i$ denote the phase voltage and the phase current, $R$ is the coil resistance, $L$ is the self-inductance, $M$ is the mutual inductance, $e$ is the back electromotive force of each phase.

Since each phase coil is arranged symmetrically with the same number of turns, below conditions are satisfied:

$$
\begin{aligned}
& R_{u}=R_{v}=R_{w} \equiv R \cdots \cdots \\
& L_{u u}=L_{v v}=L_{w w} \cdots \cdots \\
& M_{u v}=M_{v w}=M_{w u} \equiv M .
\end{aligned}
$$

From Eqs. (15)-(17), electrical dynamics of the proposed actuator can be defined as:

$$
\left[\begin{array}{c}
v_{u} \\
v_{v} \\
v_{w}
\end{array}\right]=\left[\begin{array}{ccc}
R+\rho L & 0 & 0 \\
0 & R+\rho L & 0 \\
0 & 0 & R+\rho L
\end{array}\right]\left[\begin{array}{c}
i_{u} \\
i_{v} \\
i_{w}
\end{array}\right]+\left[\begin{array}{c}
e_{u} \\
e_{v} \\
e_{w}
\end{array}\right] \ldots \ldots \ldots
$$

where $\rho=\mathrm{d} / \mathrm{dt}, L=L_{u u}-M$. It is a relational expression of leakage inductance, self-inductance, and mutual inductance. As a result, the electrical dynamics of the proposed actuator is the same as that of a typical surface permanent magnet synchronous motor (SPMSM).

3.2 Mechanical Dynamics The mechanical equation of two-DOF RA without mechanical spring can be expressed as follows:

$$
\left[\begin{array}{c}
\dot{x} \\
\ddot{x} \\
\dot{y} \\
\ddot{y}
\end{array}\right]=\left[\begin{array}{cccc}
0 & 1 & 0 & 0 \\
0 & -\frac{c}{m} & 0 & 0 \\
0 & 0 & 0 & 1 \\
0 & 0 & 0 & -\frac{c}{m}
\end{array}\right]\left[\begin{array}{c}
x \\
\dot{x} \\
y \\
\dot{y}
\end{array}\right]+\frac{1}{m}\left[\begin{array}{c}
0 \\
F_{e x}-F_{d x}-F_{l x} \\
0 \\
F_{e y}-F_{d y}-F_{l y}
\end{array}\right]
$$

where $x$ is the displacement in the $X$-direction, $y$ is the displacement in the $Y$-direction, $m$ denotes the mass of the mover, $c$ means a viscosity coefficient considering the support structure of the mover, $F_{\text {ex,ey }}$ is the force generated by the current, $F_{d x, d y}$ means the centering force by the detent, $F_{l x, l y}$ is the force of the load.

In addition, the resonant frequency of the two-DOF RA can be defined as follows:

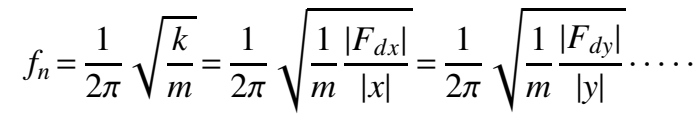

where $k$ is the stiffness of the mechanical spring. However, since the proposed actuator has no mechanical spring, the resonant frequency of the proposed actuator is determined by the ratio between the amplitude of detent force and displacement.

\section{Motion Estimation}

The electrical dynamics of the proposed actuator in $X-Y$ orthogonal reference frame can be defined as:

$$
\left[\begin{array}{l}
v_{x} \\
v_{y}
\end{array}\right]=\left[\begin{array}{cc}
R+\rho L & 0 \\
0 & R+\rho L
\end{array}\right]\left[\begin{array}{l}
i_{x} \\
i_{y}
\end{array}\right]+K_{e}\left[\begin{array}{c}
\dot{y} \\
-\dot{x}
\end{array}\right] \text {. }
$$

where $K_{e}$ is the back-EMF constant. From the electrical dynamics, position and angle information can be estimated as:

$$
\begin{aligned}
& {\left[\begin{array}{l}
x \\
y
\end{array}\right]=\frac{1}{K_{e}}\left[\int\left(-v_{y}+R i_{y}\right) d t+L i_{y}\right.} \\
& {\left[\begin{array}{l}
x_{\theta} \\
y_{\theta}
\end{array}\right]=\left[\begin{array}{cc}
\cos \theta & \sin \theta \\
-\sin \theta & \cos \theta
\end{array}\right]\left[\begin{array}{l}
x \\
y
\end{array}\right] \ldots \ldots}
\end{aligned}
$$

where $x_{\theta}$ and $y_{\theta}$ are the represented position in the orthogonal frame rotated by an angle $\theta$. In addition, stroke of motion can be obtained as follows:

$$
\left[\begin{array}{l}
\alpha \\
\beta
\end{array}\right]=\left[\begin{array}{l}
\max \left(x_{\theta}\right)-\min \left(x_{\theta}\right) \\
\max \left(y_{\theta}\right)-\min \left(y_{\theta}\right)
\end{array}\right] .
$$

where $\alpha$ and $\beta$ are the stroke of motion. Figure 4 shows a type of elliptical motion. If elliptical motion can be controlled, scrolling motion and reciprocating motion can be performed by changing the ratio between $\alpha$ and $\beta$. 


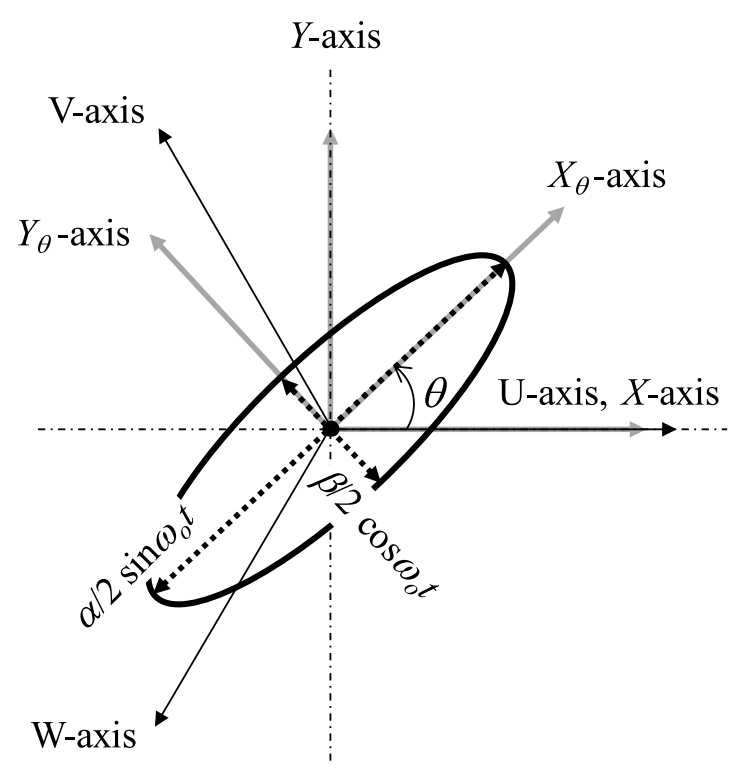

Fig. 4. Definition of elliptical motion in the reference frame

\section{Verification through FEA}

FEA is performed to confirm the trend of parameter characteristics such as the direction of the detent and the thrust in all of the mover position. Dense meshes are only applied in the surface of the teeth-tip and permanent magnet due to the reduction of computation time. In addition, 441 cases of mover positions are analyzed by positioning the mover within a range of $4 \mathrm{~mm}$ in $X-Y$ plane. As an analysis tool, JMAG software is used.

Figure 5 presents the detent characteristics of the proposed actuator. The degree of the brightness means the norm of the detent, arrows are pointing at a direction of detent force. We confirm that contours are drawn with regular circles in $X-Y$ plane and all of the arrows are pointing at the origin. As a result, detent can be used as stiffness for resonance with twoDOF.

The result of the thrust characteristics is shown in Fig. 6. In Fig. 6(a), each phase current is set with $i_{u}=0 \mathrm{~A}, i_{v}=0.58 \mathrm{~A}$, $i_{w}=-0.58 \mathrm{~A}$. All of the arrows mean the directions of the thrust and they are pointing at $X$-direction. Figure $6(\mathrm{~b})$ shows

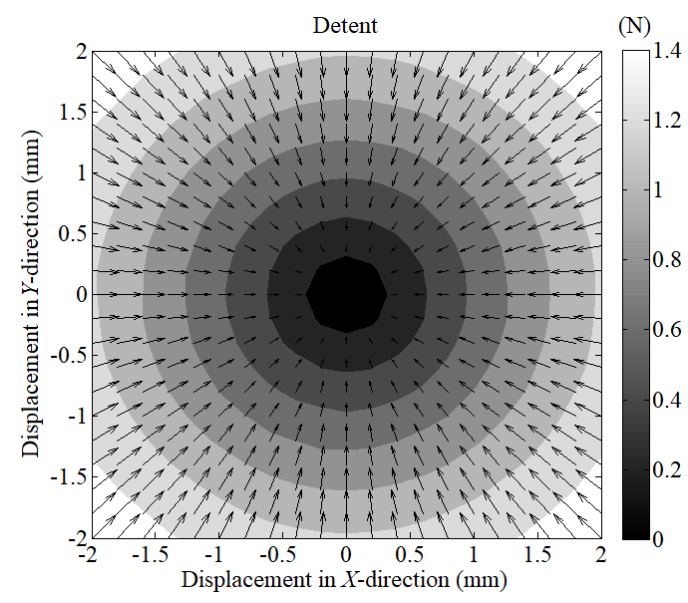

Fig. 5. Results of detent characteristics

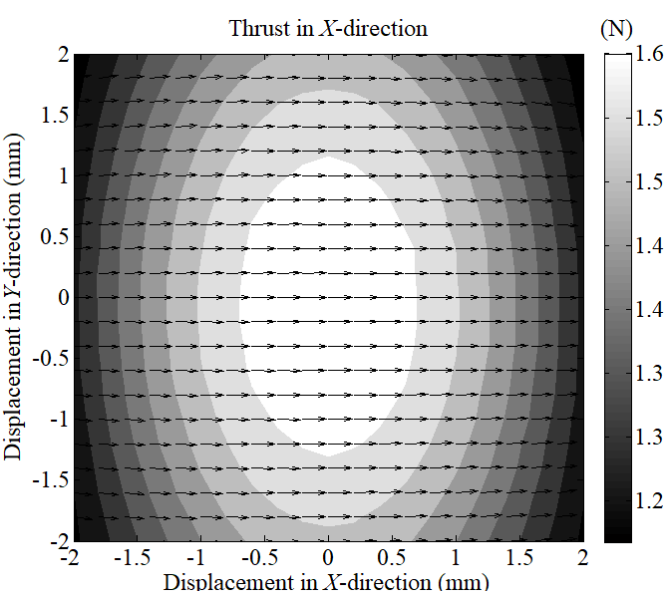

(a)

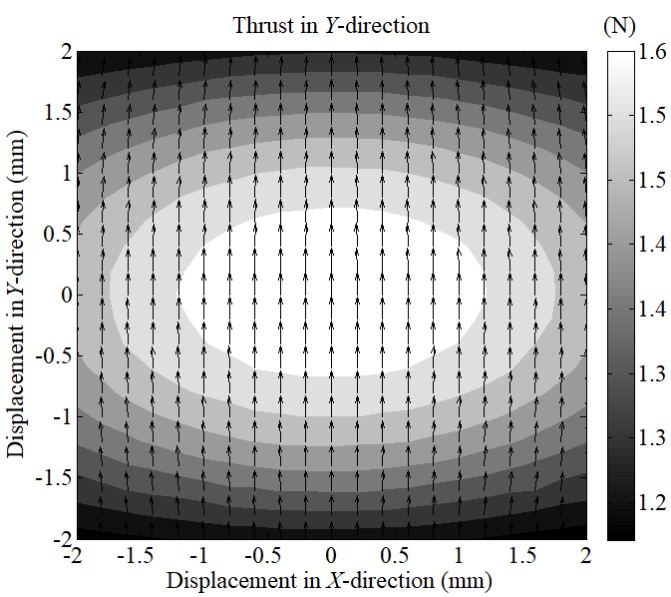

(b)

Fig. 6. Results of thrust characteristics. (a) $X$-direction (b) $Y$-direction

the result of thrust in $Y$-direction. To generate the thrust in $Y$ direction, each phase current is set with $i_{u}=-0.67 \mathrm{~A}, i_{v}=$ $0.33 \mathrm{~A}, i_{c}=0.33 \mathrm{~A}$. All of the arrows keep in $Y$-direction.

\section{Experimental Verification}

Figure 7 present the prototype of the proposed two-DOF RA. In order to support the mover and limit the stroke, stator guide, mover stopper, spacer, ball stopper, ball-bearing were added. The specification of the prototype is listed in Table 1.

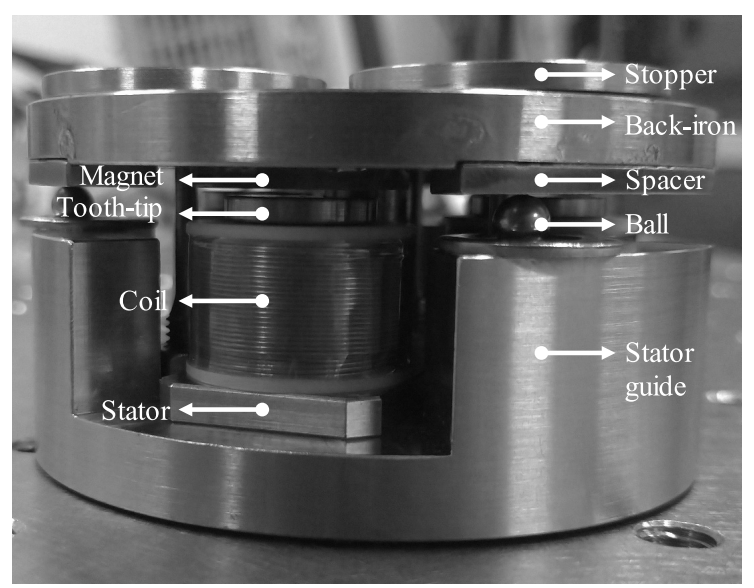

Fig. 7. Prototype of the proposed two-DOF RA 
Table 1. Model specification

\begin{tabular}{cc}
\hline \hline Parameter & Value \\
\hline Mass of mover $(\mathrm{g})$ & 66.1 \\
Effective stiffness of detent $(\mathrm{N} / \mathrm{m})$ & 540 \\
Force constant $(\mathrm{N} / \mathrm{A})$ & 1.67 \\
Back-EMF constant $(\mathrm{Vs} / \mathrm{m})$ & 1.67 \\
Phase resistance $(\Omega)$ & 4.1 \\
Phase inductance $(\mathrm{mH})$ & 15.7 \\
Resonant frequency $(\mathrm{Hz})$ & 14.9 \\
\hline \hline
\end{tabular}

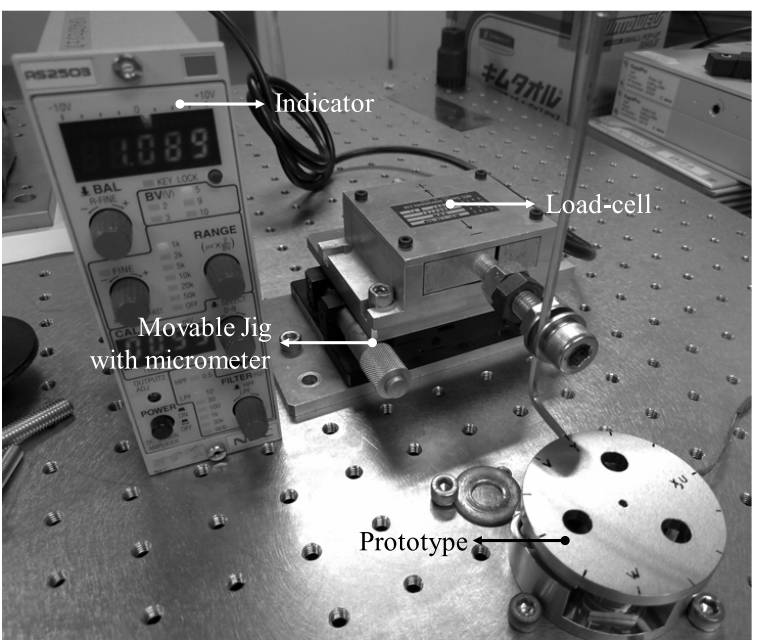

Fig. 8. Setup for measuring the static characteristics

6.1 Detent Measurement Figure 8 shows the setup environment for measuring the static characteristics of the prototype. It consists of a load cell that can move in one direction through a micrometer and the indicator that measures the output signal of the load cell.

Figure 9 illustrates the results of detent characteristics. Figure 9(a) is the result in the $X$-direction, and Fig. 9(b) is the result in the $Y$-direction. The lines mean the result of FEA, and dots denote the result of measurement. The slopes of the measured values of the detent in the $X$ and $Y$ directions are $540 \mathrm{~N} / \mathrm{m}$ and $543 \mathrm{~N} / \mathrm{m}$, indicating an error within $0.6 \%$. On the other hand, the result of FEA is $576 \mathrm{~N} / \mathrm{m}$, which is $6 \%$ higher than the measured value. This is due to the fact that the air-gap length may be increased by assembly tolerances. As a result, the resonant frequency is lower than the expected value, but it is possible to realize the two-DOF resonant motion.

6.2 Force Constant Measurement Figure 10 denotes the result of force constant characteristics. To generate thrust in the $Y$ direction, the phase current is energized as switching-states $(0,1,1)$ in Fig. 3 . The measured value is about $2 \%$ smaller than the result of FEA. This is also due to the fact that the air-gap length is increased. As a result, force constant is about 1.67 N/A in the moving distance within $2 \mathrm{~mm}$.

6.3 Inductance and Resistance Measurement From (16), to estimate the motion of the mover, we have to know the information about inductance and resistance. However,

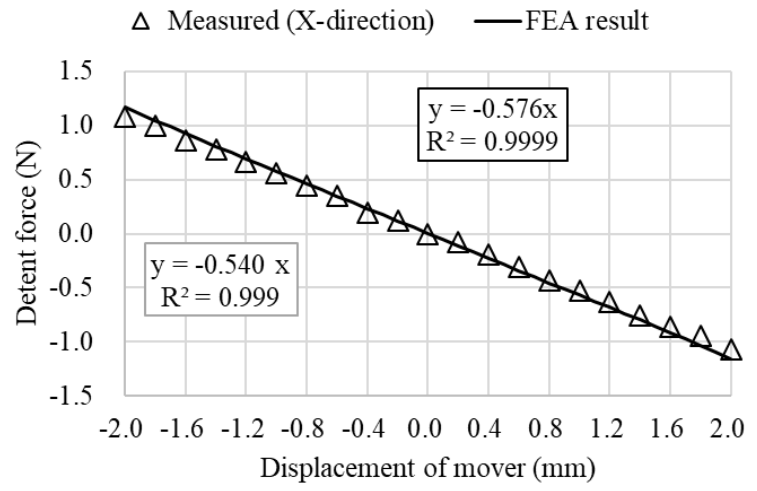

(a)

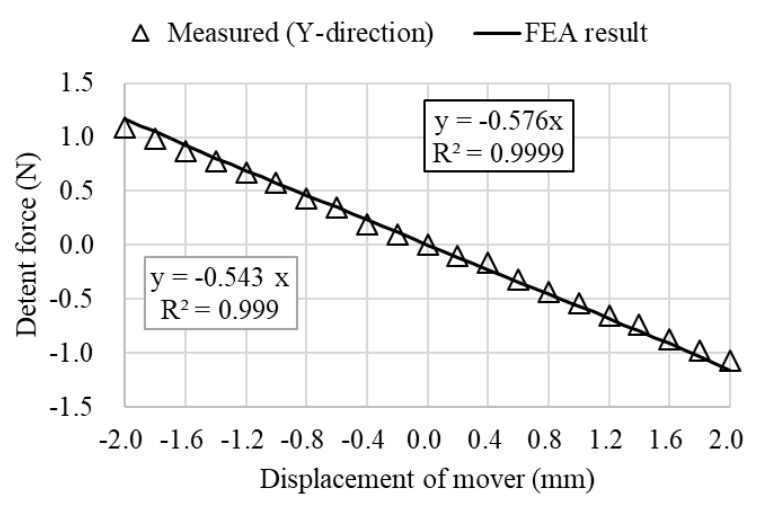

(b)

Fig. 9. Result of detent characteristics. (a) detent in the $X$-direction (b) detent in the $Y$-direction

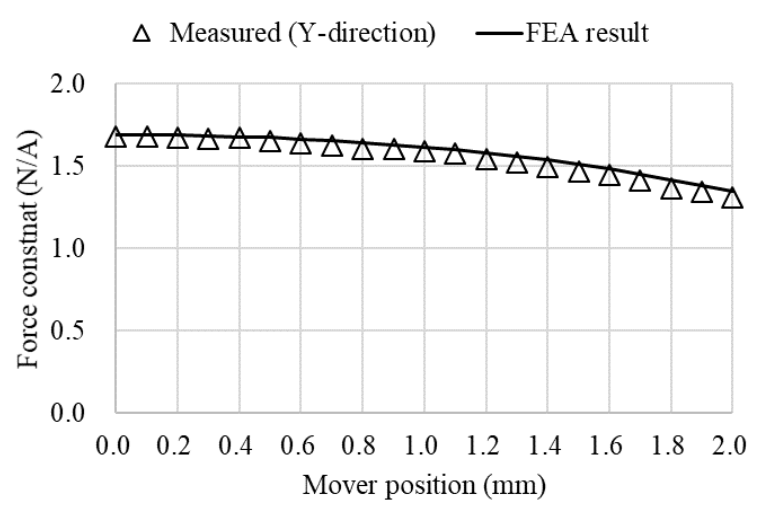

Fig. 10. Result of force constant characteristics

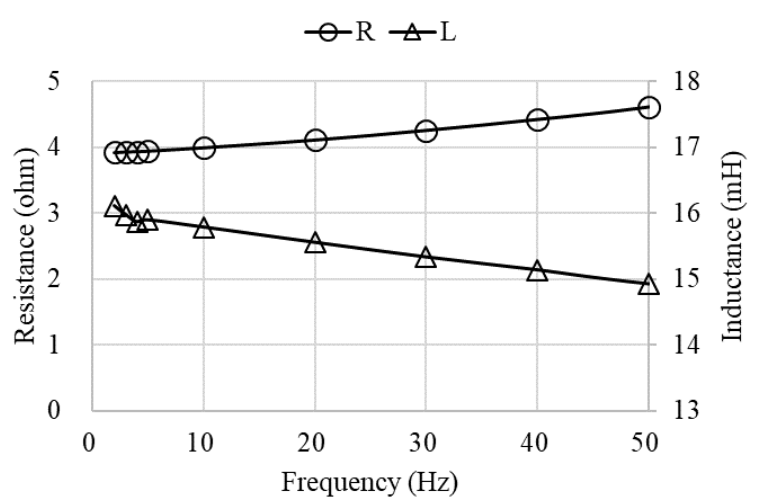

Fig. 11. Result of inductance and resistance 


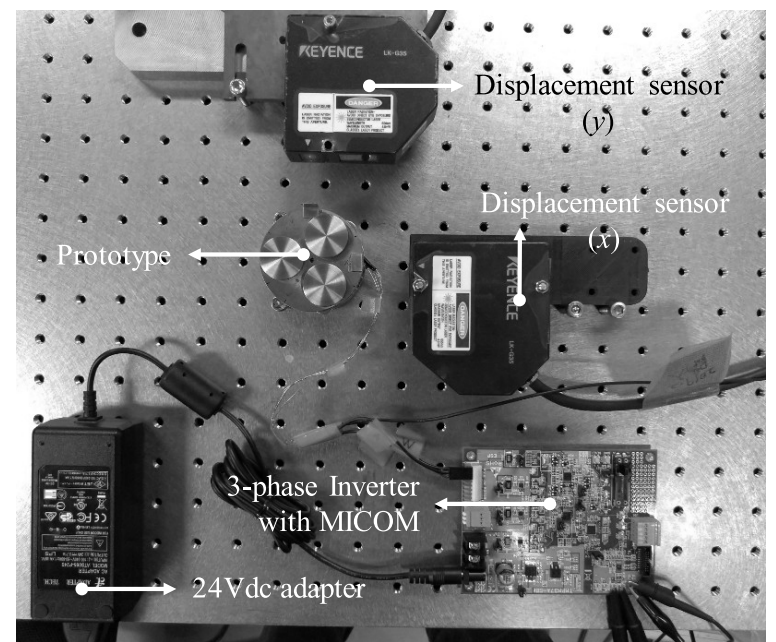

Fig. 12. Setup for measuring the dynamic characteristics

Table 2. Operation condition

\begin{tabular}{cc}
\hline \hline Parameter & Value \\
\hline Operating frequency $(\mathrm{Hz})$ & 15.0 \\
Carrier frequency $(\mathrm{kHz})$ & 4.0 \\
DC-link voltage $(\mathrm{V})$ & 24 \\
Target rotation angle (degree) & 45 \\
Target stroke (mm) & 2.0 \\
Ratio of reciprocating motion $(\beta / \alpha)$ & 0 \\
Ratio of elliptical motion $(\beta / \alpha)$ & 0.5 \\
Ratio of scrolling motion $(\beta / \alpha)$ & 1.0 \\
\hline \hline
\end{tabular}

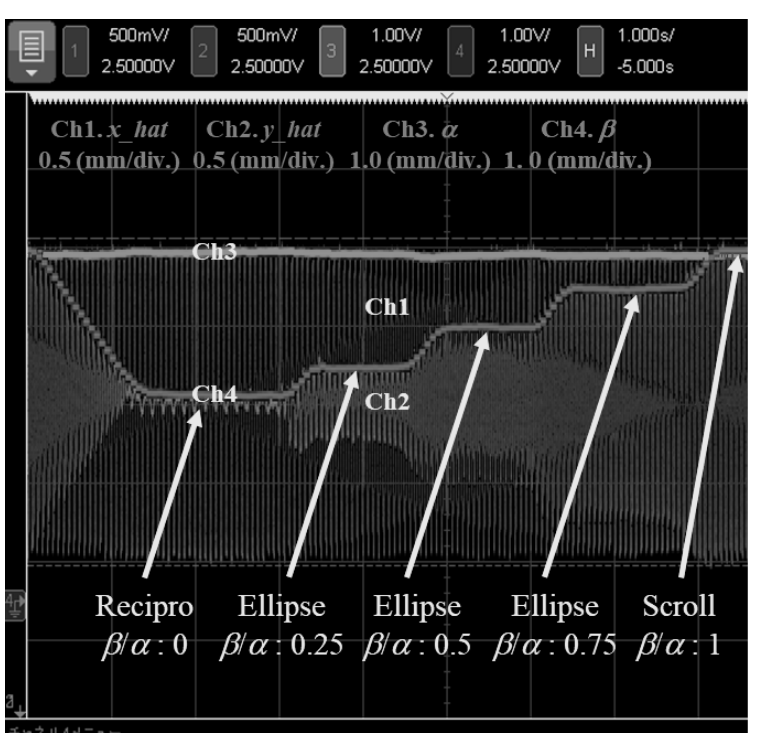

Fig. 13. DAC outputs in oscilloscope

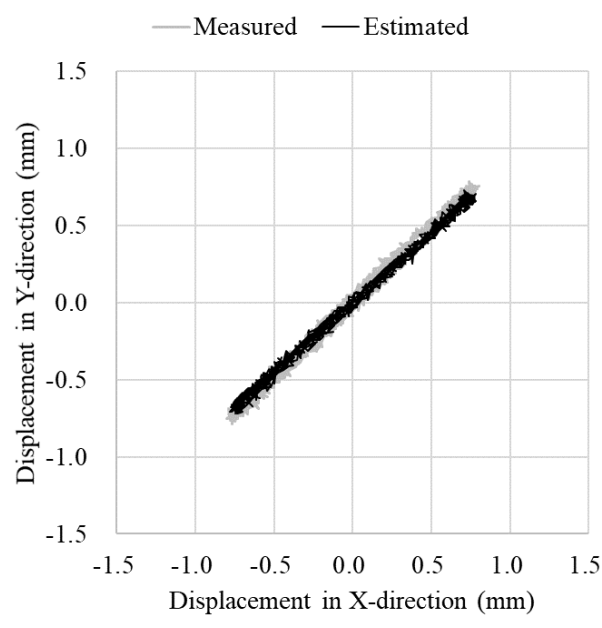

(a)

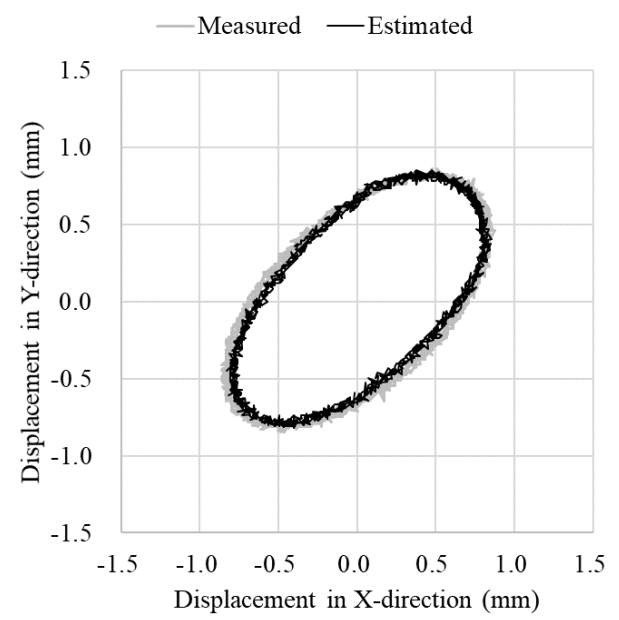

(b)

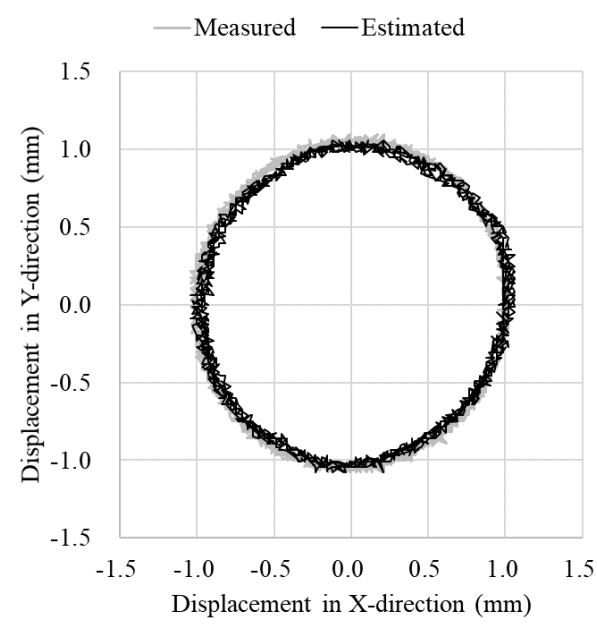

(c)

Fig. 14. Results of the comparison between measured and estimated displacement. (a) reciprocating (b) ellipse (c) scroll

by operating frequency, their impedance may be changed due to the component of eddy-current and hysteresis loss. Figure 11 denotes the result of inductance and resistance in the frequency domain. Since we know that the operating frequency is about $15 \mathrm{~Hz}$, we decide the value of inductance and resistance for motion estimation as $15.7 \mathrm{mH}$ and $4.1 \Omega$.

6.4 Motion Control Figure 12 describes the setup 


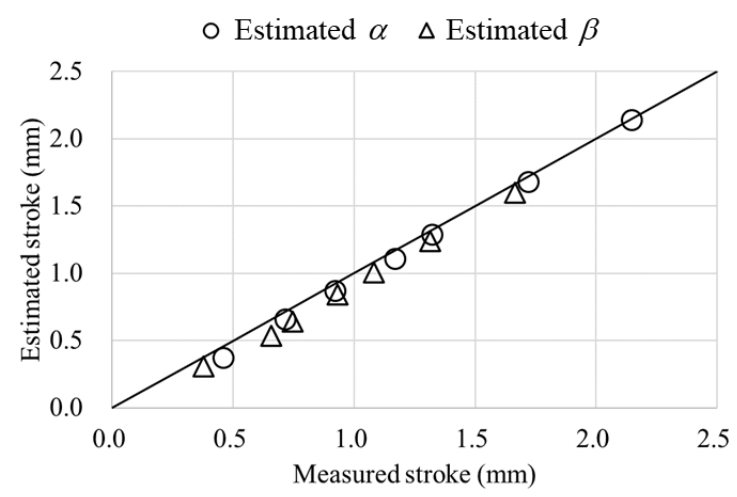

Fig. 15. Result of stroke estimation on the elliptical motion

environment for measuring the dynamic characteristics of the prototype. It consists of the prototype, position sensor, oscilloscope, and three-phase inverter. Since the inverter has fourchannel of digital to analog converter (DAC), measured value and estimated value can be compared under the oscilloscope. The operation condition is presented in Table 2.

Figure 13 shows the result of changing the motion. Each channel of the oscilloscope denotes DAC outputs of $x, y, \alpha$, and $\beta$. We confirmed that reciprocating motion, elliptical motion, scrolling motion can be realized by controlling the ratio of $\alpha$ and $\beta$.

Figure 14 illustrates the result of the comparison between the measured displacement and the estimated displacement. Figure 14(a) indicates the result of displacement trajectory for reciprocating motion. As indicated in the figure, reciprocating motion in a direction can be controlled as well. Figure 14(b) denotes the result of elliptical motion. It can be confirmed that all of the command motion, measured motion, and estimated motion match successfully. Finally, the result of scrolling motion is shown in Fig. 14(c). As a result, we proved that all of the motion can be estimated and controlled as well.

Figure 15 shows the result of the comparison between the measured stroke and the estimated stroke in the elliptical motion. By using the measured parameters in the stroke estimation process, a good result of estimation accuracy can be obtained.

\section{Conclusion}

In this study, the authors have proposed an axial gap type two-DOF RA which resonates without a mechanical spring. The proposed two-DOF RA showed that constant detent force is generated with respect to the moving direction from theoretical and experimental results. Furthermore, a method of estimating the motion of an actuator without a position sensor has been proposed. We have built the prototype to experimentally verify the effectiveness of the proposed method. The static characteristics of the prototype closed to the result of theoretic and FEA. In addition, we designed an estimator and controller in the microcomputer (MICOM) of the inverter. Finally, it has been confirmed that various resonant motion can be precisely estimated and controlled as well.

\section{References}

( 1 ) I. Boldea and S.A. Nasar: "Linear electric actuators and generators", IEEE Transactions on Energy Conversion, Vol.14, No.3, pp.712-717 (1999)

( 2 ) J. Wang, D. Howe, and Z. Lin: "Comparative Studies on Linear Motor Topologies for Reciprocating Vapor Compressors”, 2007 IEEE International Electric Machines \& Drives Conference, Antalya, pp.364-369 (2007)

( 3 ) D. Ebihara and M. Watada: "Development of a single-winding linear oscillatory actuator (artificial heart application)", IEEE Transactions on Magnetics, Vol.28, No.5, pp.3030-3032 (1992)

( 4 ) Y. Asai, K. Hirata, and T. Ota: "Amplitude control method of linear resonant actuator by load estimation from the back-EMF", IEEE Transactions on Magnetics, Vol.49, No.5, pp.2253-2256 (2013)

( 5 ) Z. Zhu, X. Chen, D. Howe, and S. Iwasaki: "Electromagnetic modeling of a novel linear oscillating actuator", IEEE Transactions on Magnetics, Vol.44, No.11, pp.3855-3858 (2008)

( 6 ) K. Hirata, T. Yamamoto, T. Yamaguchi, Y. Kawase, and Y. Hasegawa: "Dynamic analysis method of two-dimensional linear oscillatory actuator employing finite element method", IEEE Transactions on Magnetics, Vol.43, No.4, pp.1441-1444 (2007)

( 7 ) Y. Kono, T. Yoshimoto, and K. Hirata: "Characteristics analysis of a haptic device using a 2-DOF linear oscillatory actuator", International Journal of Applied Electromagnetics and Mechanics, Vol.45, No.1-4, pp.909-916 (2014)

( 8 ) G. Kim and K. Hirata: "Motion Control of a Two-Degree-of-Freedom Linear Resonant Actuator without a Mechanical Spring", Sensors, Vol.20, No.7, p.1954 (2020)

( 9 ) G. Kim and K. Hirata: "Motion Control of Two-Degree-of-Freedom Linear Resonant Actuator", Linear Drives Technical Committee, LD-19-006, pp.29_ 34 (2020)

Gyunam Kim (Non-member) received the B.S. and M.S. degrees

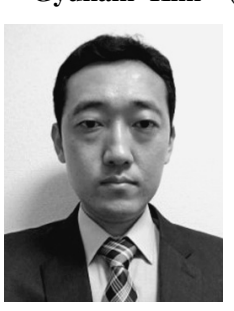
from Niigata University, Japan, in 2005 and 2007, respectively. He was researcher at the R\&D lab., LG Electronics, Korea, from 2007 to 2017. He has been engaged in research of electrical machines in the Department of Adaptive Machine Systems, Graduate School of Engineering at Osaka University, Japan. He has received Best Presentation Award (APSAEM) in 2018. He received COMPEL Best Paper Award (ISEF) in 2019.

Katsuhiro Hirata (Senior Member) received the B.E. degree from

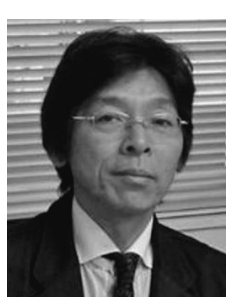
Osaka University in 1982 and D.E. degree from Doshisha University in 1996, respectively. He was a researcher at the R\&D lab., Matsushita Electric Works Ltd. from 1982 to 2005 . He jointed Osaka University in 2005. He is presently a professor in the Department of Adaptive Machine Systems, Graduate School of Engineering at Osaka University. He has been engaged in research of electromagnetic applied actuators and sensors. He has received Ministry Award of Education, Science \& Technology (Ministry of Education, Culture, Sports, Science and Technology-Japan) in 2003. IEEE member. He has received OHM Technology Award (Promotion Foundation for Electrical Science and Engineering) in 2004. He received Advanced Technology Award and Paper Award (IEEJ) in 2007, 2009. 